\title{
On multivalued iteration semigroups
}

\author{
Magdalena PisZCZEK
}

\begin{abstract}
We will give a necessary and sufficient condition for the family $\left\{F_{t}: t \geq 0\right\}$ of multifunctions $F_{t}(x)=\sum_{i=0}^{\infty} \frac{t^{i}}{i !} G^{i}(x)$, where $G$ is a continuous and additive multifunction, to be an iteration semigroup.
\end{abstract}

Mathematics Subject Classification (2000). 26E25, 39B12, 47D03.

Keywords. Iteration semigroup, Hukuhara's derivative, Riemann integral of multifunctions.

In the paper Smajdor [10] showed that the condition

$$
G+t G^{2}=(I+t G) \circ G, \quad t \geq 0,
$$

where $I$ is the identical map and $\circ$ denotes superposition, is a necessary and sufficient condition under which the family $\left\{F_{t}: t \geq 0\right\}$ of multifunctions $F_{t}(x)=\sum_{i=0}^{\infty} \frac{t^{i}}{i !} G^{i}(x)$, where $G$ is a continuous and additive multifunction, is an iteration semigroup, with the assumption $0 \in G(x)$ for all $x \in K$. We want to present another one without the assumption that $0 \in G(x)$ and its correlation.

Throughout this paper all vector spaces are supposed to be real. Let $X$ be a vector space. We define

$$
A+B:=\{a+b: a \in A, b \in B\}, \quad t A:=\{t a: a \in A\},
$$

where $A, B \subset X$ and $t \in \mathbb{R}$.

A subset $K$ of $X$ is called a cone if $t K \subset K$ for all positive $t$. A cone is said to be convex if it is a convex set.

Let $X$ and $Y$ be two vector spaces and let $K \subset X$ be a convex cone. A setvalued function $F: K \rightarrow n(Y)$, where $n(Y)$ denotes the family of all nonempty subsets of $Y$, is called additive if

$$
F(x+y)=F(x)+F(y)
$$


for all $x, y \in K$. If additionally $F$ satisfies

$$
F(t x)=t F(x)
$$

for all $x \in K$ and $t \geq 0$, then $F$ is linear.

Let $c(Y)$ denote the family of all nonempty compact subsets of a normed space $Y$. The continuity of a multifunction with compact values denotes continuity with respect to the Hausdorff metric $d$.

Let $X, Y$ be normed spaces and $K$ be a closed convex cone in $X$. The norm $\|F\|$ of a continuous additive multifunction $F: K \rightarrow n(Y)$ is the smallest element of the set $\{M>0:\|F(x)\| \leq M\|x\|, x \in K\}$.

Lemma 1. Let $K$ be a closed convex cone with a nonempty interior in a Banach space and let $Y$ be a normed space. Suppose that $F_{n}: K \rightarrow c(Y), n \in \mathbb{N}$, are continuous additive set-valued functions. If

$$
\lim _{n \rightarrow \infty} F_{n}(x)=F(x) \quad \text { for } x \in K,
$$

then $F$ is continuous and additive.

Proof. It is clear that $F$ is linear.

As $\left(F_{n}\right)_{n \in \mathbb{N}}$ is convergent to $F$, the set

$$
\bigcup_{n=1}^{\infty} F_{n}(x)
$$

is bounded for every $x \in K$. By Theorem 3 in Ref. [12] there exists a positive constant $M$ such that

$$
\left\|F_{n}\right\| \leq M \quad \text { for } n=1,2, \ldots .
$$

Moreover, by Lemma 5 in Ref. [11] there exists $M_{0}>0$ such that

$$
d\left(F_{n}(x), F_{n}(y)\right) \leq M_{0}\left\|F_{n}\right\|\|x-y\|, \quad x, y \in K .
$$

Let $\epsilon>0, x, y \in K$ and $\|x-y\|<\frac{\epsilon}{3 M_{0} M}$. There exists $n \in \mathbb{N}$ such that

Thus, by (1) and (2) we have

$$
d\left(F(x), F_{n}(x)\right)<\frac{\epsilon}{3} \quad \text { and } \quad d\left(F(y), F_{n}(y)\right)<\frac{\epsilon}{3} .
$$

$$
\begin{aligned}
d(F(x), F(y)) & \leq d\left(F(x), F_{n}(x)\right)+d\left(F_{n}(x), F_{n}(y)\right)+d\left(F_{n}(y), F(y)\right) \\
& <\frac{\epsilon}{3}+\frac{\epsilon}{3}+\frac{\epsilon}{3}=\epsilon .
\end{aligned}
$$

This means that $F$ is continuous.

Lemma 2. (Lemma 7 in Ref. [6]) Let $K$ be a closed convex cone with a nonempty interior in a Banach space and let $Y$ be a normed space. Suppose that $F, F_{n}: K \rightarrow c(Y)$ are continuous additive set-valued functions. If

$$
\lim _{n \rightarrow \infty} F_{n}(x)=F(x) \quad \text { for } x \in K,
$$

then the sequence $\left(F_{n}\right)_{n \in \mathbb{N}}$ uniformly converges to $F$ on every $D \in c(K)$. 
Lemma 3. (Lemma 4 in Ref. [8]) Let $D$ be a nonempty set and $Y$ be a normed space. If $F, F_{n}: D \rightarrow c(Y)$ are set-valued functions and the sequence $\left(F_{n}\right)_{n \in \mathbb{N}}$ uniformly converges to $F$ on $D$, then

$$
\lim _{n \rightarrow \infty} F_{n}(D)=F(D) \text {. }
$$

Lemma 4. (Theorem 2 in Ref. [2]) Let $\left(X, \rho_{X}\right)$ and $\left(Y, \rho_{Y}\right)$ be two metric spaces and let $d_{X}$ and $d_{Y}$ be the Hausdorff metrics derived from $\rho_{X}$ and $\rho_{Y}$, respectively. If $F: X \rightarrow n(Y)$ is a set-valued function and $M$ is a positive constant such that

$$
d_{Y}(F(x), F(y)) \leq M \rho_{X}(x, y)
$$

for all $x, y \in X$, then

$$
d_{Y}(F(A), F(B)) \leq M d_{X}(A, B)
$$

for every nonempty subsets $A, B$ of $X$.

The superposition $G \circ F$ of two multifunctions $F, G: K \rightarrow n(K)$ is defined as follows

$$
(G \circ F)(x)=G(F(x))=\bigcup_{y \in F(x)} G(y) .
$$

Lemma 5. Let $K$ be a closed convex cone with a nonempty interior in a Banach space. Suppose that $F_{n}, F, G_{n}, G: K \rightarrow c(K), n \in \mathbb{N}$, are continuous additive set-valued functions. If $\lim _{n \rightarrow \infty} F_{n}(x)=F(x)$ and $\lim _{n \rightarrow \infty} G_{n}(x)=G(x)$ for $x \in K$, then

$$
\lim _{n \rightarrow \infty} F_{n}\left(G_{n}(x)\right)=F(G(x))
$$

for $x \in K$.

Proof. Fix $x \in K$. From Lemma 5 in Ref. [11] and Lemma 4 there exists $M_{0}>0$ such that

$$
\begin{aligned}
d\left(F_{n}\left(G_{n}(x)\right), F(G(x))\right) & \leq d\left(F_{n}\left(G_{n}(x)\right), F_{n}(G(x))\right)+d\left(F_{n}(G(x)), F(G(x))\right) \\
& \leq M_{0}\left\|F_{n}\right\| d\left(G_{n}(x), G(x)\right)+d\left(F_{n}(G(x)), F(G(x))\right) .
\end{aligned}
$$

By the same argument as in the proof of Lemma 1 there exists a positive constant $M$ such that

$$
\left\|F_{n}\right\| \leq M \quad \text { for } n=1,2, \ldots
$$

Moreover, by Lemma 2 , the sequence $\left(F_{n}\right)_{n \in \mathbb{N}}$ is uniformly convergent to $F$ on every nonempty compact subset of $K$. Thus, from Lemma 3 ,

$$
\lim _{n \rightarrow \infty} d\left(F_{n}\left(G_{n}(x)\right), F(G(x))\right)=0 .
$$

Let $K$ be a convex cone in a normed space and let $c c(K)$ stand for the family of all compact convex members of $n(K)$. The Hukuhara difference $A-B$ 
of $A, B \in c c(K)$ is a set $C \in c c(K)$ such that $A=B+C$. By Rådström's Cancellation Lemma [5] it follows that if this difference exists, then it is unique.

Lemma 6. (Lemma 3 in Ref. [7]) Let $X$ and $Y$ be two normed vector spaces and let $K$ be a closed convex cone in $X$. Assume that $F: K \rightarrow c c(K)$ is a continuous additive set-valued function and $A, B \in c c(K)$. If there exists the difference $A-B$, then there exists $F(A)-F(B)$ and $F(A)-F(B)=F(A-B)$.

Let $F, G: K \rightarrow c c(K)$. We can define the multifunctions $F+G$ and $F-G$ on $K$ as follows

$$
(F+G)(x):=F(x)+G(x) \text { for } x \in K
$$

and

$$
(F-G)(x):=F(x)-G(x)
$$

if the Hukuhara differences $F(x)-G(x)$ exist for all $x \in K$.

Lemma 7. (Lemma 2 in Ref. [4]) For each set $A \subset K$ the inclusion

$$
(F+G)(A) \subset F(A)+G(A)
$$

holds. Moreover, if there exist the Hukuhara difference $F(A)-G(A)$ and the multifunction $F-G$, then

$$
F(A)-G(A) \subset(F-G)(A) .
$$

For a multifunction $F:[a, b] \rightarrow c c(X)$ such that there exist the Hukuhara differences $F(t)-F(s)$ as $a \leq s \leq t \leq b$, the Hukuhara derivative at $t \in(a, b)$ is defined by the formula

$$
D F(t)=\lim _{h \rightarrow 0^{+}} \frac{F(t+h)-F(t)}{h}=\lim _{h \rightarrow 0^{+}} \frac{F(t)-F(t-h)}{h},
$$

whenever both of these limits exist with respect to the Hausdorff metric $d$ in $c c(K)$ derived from the norm in $X$ (see Ref. [1]). Moreover,

$$
D F(a)=\lim _{s \rightarrow a^{+}} \frac{F(s)-F(a)}{s-a}, \quad D F(b)=\lim _{s \rightarrow b^{-}} \frac{F(b)-F(s)}{b-s} .
$$

Lemma 8. (Lemma 5 in Ref. [9]) If $F, G:[a, b] \rightarrow c c(X)$ are two differentiable multifunctions such that $D F(t)=D G(t)$ for $t \in[a, b]$ and $F(a)=G(a)$, then

$$
F(t)=G(t) \text { for } t \in[a, b] .
$$

Let $X$ be a Banach space and let $[a, b] \subset \mathbb{R}$. If a multifunction $F:[a, b] \rightarrow$ $c c(X)$ is continuous, then there exists the Riemann integral of $F$ (see Ref. [1]). We need the following properties of the Riemann integral.

Lemma 9. ([1] p. 211) If $F:[a, b] \rightarrow c c(X)$ is continuous, then

$$
\left\|\int_{a}^{b} F(t) d t\right\| \leq \int_{a}^{b}\|F(t)\| d t .
$$


Lemma 10. (Lemma 7 in Ref. [8]) Let $K$ be a convex cone in $X$. If $F: K \rightarrow$ $c c(X)$ is continuous and additive, $G:[a, b] \rightarrow c c(K)$ is continuous, then

$$
\int_{a}^{b} F(G(t)) d t=F\left(\int_{a}^{b} G(t) d t\right)
$$

Lemma 11. (Lemma 4 in Ref. [11]) If $F:[a, b] \rightarrow c c(X)$ is continuous and $H(t)=\int_{a}^{t} F(u) d u$, then $D H(t)=F(t)$ for $a \leq t \leq b$.

Lemma 12. If $F:[0,+\infty) \rightarrow c c(X)$ is continuous, then

$$
\int_{0}^{t}\left(\frac{(t-u)^{n}}{n !} \int_{0}^{u} F(s) d s\right) d u=\int_{0}^{t} \frac{(t-u)^{n+1}}{(n+1) !} F(u) d u
$$

for $t \geq 0$ and $n=0,1, \ldots$

Proof. For every nonnegative integer $n$ we define

$$
\phi_{n}(t)=d\left(\int_{0}^{t}\left(\frac{(t-u)^{n}}{n !} \int_{0}^{u} F(s) d s\right) d u, \int_{0}^{t} \frac{(t-u)^{n+1}}{(n+1) !} F(u) d u\right), \quad t \geq 0 .
$$

For $n=0$

$$
\phi_{0}(t)=d\left(\int_{0}^{t}\left(\int_{0}^{u} F(s) d s\right) d u, \int_{0}^{t}(t-u) F(u) d u\right), \quad t \geq 0
$$

and according to Lemma 12 in Ref. [3], we have $\phi_{0} \equiv 0$.

Fix $n \in \mathbb{N}$ and we suppose that $\phi_{i} \equiv 0$ for all $i \leq n$. By the properties of the Riemann integral we obtain, for $0<k<1$, the following equalities

$$
\begin{aligned}
& \int_{0}^{t+k}\left(\frac{(t+k-u)^{n+1}}{(n+1) !} \int_{0}^{u} F(s) d s\right) d u \\
& =\int_{0}^{t}\left(\frac{(t-u)^{n+1}}{(n+1) !} \int_{0}^{u} F(s) d s\right) d u+\sum_{i=1}^{n+1} \frac{k^{i}}{i !} \int_{0}^{t}\left(\frac{(t-u)^{n+1-i}}{(n+1-i) !} \int_{0}^{u} F(s) d s\right) d u \\
& \quad+\int_{t}^{t+k}\left(\frac{(t+k-u)^{n+1}}{(n+1) !} \int_{0}^{u} F(s) d s\right) d u
\end{aligned}
$$


and

$$
\begin{aligned}
& \int_{0}^{t+k} \frac{(t+k-u)^{n+2}}{(n+2) !} F(u) d u \\
& \quad=\int_{0}^{t} \frac{(t-u)^{n+2}}{(n+2) !} F(u) d u \\
& \quad+\sum_{i=1}^{n+2} \frac{k^{i}}{i !} \int_{0}^{t} \frac{(t-u)^{n+2-i}}{(n+2-i) !} F(u) d u+\int_{t}^{t+k} \frac{(t+k-u)^{n+2}}{(n+2) !} F(u) d u .
\end{aligned}
$$

Using the last equality and the induction assumption we conclude that

$$
\begin{aligned}
& \frac{\phi_{n+1}(t+k)-\phi_{n+1}(t)}{k} \\
& \leq\left\|\frac{1}{k} \int_{t}^{t+k}\left(\frac{(t+k-u)^{n+1}}{(n+1) !} \int_{0}^{u} F(s) d s\right) d u\right\| \\
& \quad+\left\|\frac{1}{k} \int_{t}^{t+k} \frac{(t+k-u)^{n+2}}{(n+2) !} F(u) d u\right\|+\left\|\int_{0}^{t} \frac{k^{n+1}}{(n+2) !} F(u) d u\right\| .
\end{aligned}
$$

Since

$$
\left\|\frac{1}{k} \int_{t}^{t+k}\left(\frac{(t+k-u)^{n+1}}{(n+1) !} \int_{0}^{u} F(s) d s\right) d u\right\| \leq \frac{(t+k) k^{n+1}}{(n+2) !} M
$$

and

$$
\left\|\frac{1}{k} \int_{t}^{t+k} \frac{(t+k-u)^{n+2}}{(n+2) !} F(u) d u\right\| \leq \frac{k^{n+2}}{(n+3) !} M,
$$

where $M=\sup \{\|F(s)\|: 0 \leq s \leq t+1\}$, it follows that

$$
\liminf _{k \rightarrow 0^{+}} \frac{\phi_{n+1}(t+k)-\phi_{n+1}(t)}{k} \leq 0 .
$$

Moreover, $\phi_{n+1}$ is continuous, nonnegative and $\phi_{n+1}(0)=0$, whence applying the corollary from the Zygmund Lemma we obtain $\phi_{n+1} \equiv 0$. This means that (3) holds for every $n \in \mathbb{N}$ and $t \geq 0$.

Let $K$ be a nonempty set. A family $\left\{F_{t}: t \geq 0\right\}$ of set-valued functions $F_{t}: K \rightarrow n(K)$ is said to be an iteration semigroup if

$$
F_{t} \circ F_{s}=F_{t+s}
$$

for all $t, s \geq 0$. 
Let $K$ be a convex cone in a normed space. An iteration semigroup $\left\{F_{t}\right.$ : $t \geq 0\}$ of set-valued functions $F_{t}: K \rightarrow c c(K)$ is called differentiable if all setvalued functions $t \mapsto F_{t}(x), x \in K$, have the Hukuhara derivative on $[0,+\infty)$.

Theorem 1. Let $K$ be a closed convex cone with a nonempty interior in a Banach space. If $\left\{F_{t}: t \geq 0\right\}$ is a differentiable iteration semigroup of continuous additive multifunctions $F_{t}: K \rightarrow c c(K)$ with $F_{0}(x)=\{x\}$, then

(i) $D F_{t}(x)=F_{t}(G(x))$ for all $x \in K, t \geq 0$, where $D F_{t}(x)$ denotes the Hukuhara derivarive of $F_{t}(x)$ with respect to $t$ and $G(x)=\left.D F_{t}(x)\right|_{t=0}=$ $\lim _{h \rightarrow 0^{+}} \frac{F_{h}(x)-x}{h}$ is continuous and additive,

(ii) $F_{t}(x)=x+\int_{0}^{t} F_{u}(G(x)) d u$ for all $x \in K, t \geq 0$,

(iii) For all $x \in K$ and $t \geq 0$

$$
F_{t}(x) \subset \sum_{i=0}^{\infty} \frac{t^{i}}{i !} G^{i}(x) .
$$

If additionally $F_{t} \circ G=G \circ F_{t}$ for $t \geq 0$, then

$$
F_{t}(x)=\sum_{i=0}^{\infty} \frac{t^{i}}{i !} G^{i}(x) \text {. }
$$

Proof. (i) It follows immediately from Theorem 1 in Ref. [6] and Lemma 1.

(ii) Let us fix $x \in K$. We observe, by Lemmas 2 and 3, that $t \mapsto F_{t}(G(x))$ is continuous, so it is integrable. According to (i) and Lemmas 8, 11 we get

$$
F_{t}(x)=x+\int_{0}^{t} F_{u}(G(x)) d u, \quad t \geq 0 .
$$

(iii) Let us fix $x \in K$ and $t \geq 0$. At first we show the following inclusion

$$
F_{t}(G(x)) \subset G\left(F_{t}(x)\right) .
$$

By Lemmas 2, 3, 6 and 7 we obtain

$$
\begin{aligned}
F_{t}(G(x)) & =F_{t}\left(\lim _{h \rightarrow 0^{+}} \frac{F_{h}(x)-x}{h}\right) \\
& =\lim _{h \rightarrow 0^{+}} \frac{F_{t}\left(F_{h}(x)\right)-F_{t}(x)}{h} \\
& =\lim _{h \rightarrow 0^{+}} \frac{F_{h}\left(F_{t}(x)\right)-F_{t}(x)}{h} \\
& \subset \lim _{h \rightarrow 0^{+}}\left(\frac{F_{h}-I}{h}\right)\left(F_{t}(x)\right) \\
& =G\left(F_{t}(x)\right) .
\end{aligned}
$$


From this, (ii) and Lemma 10 we have

$$
F_{t}(x)=x+\int_{0}^{t} F_{u}(G(x)) d u \subset x+G\left(\int_{0}^{t} F_{u}(x) d u\right) .
$$

If we apply (ii) to $F_{u}$ in the last inclusion, we conclude that

$$
\begin{aligned}
F_{t}(x) & \subset x+G\left(\int_{0}^{t}\left(x+\int_{0}^{u} F_{s}(G(x)) d s\right) d u\right) \\
& \subset x+t G(x)+G^{2}\left(\int_{0}^{t}\left(\int_{0}^{u} F_{s}(x) d s\right) d u\right) .
\end{aligned}
$$

Using Lemma 12 we have

$$
F_{t}(x) \subset x+t G(x)+G^{2}\left(\int_{0}^{t}(t-u) F_{u}(x) d u\right) .
$$

Repeating the same procedure we obtain

$$
\begin{gathered}
F_{t}(x) \subset x+t G(x)+\frac{t^{2}}{2 !} G^{2}(x)+\cdots+\frac{t^{n}}{n !} G^{n}(x) \\
+G^{n+1}\left(\int_{0}^{t} \frac{(t-u)^{n}}{n !} F_{u}(x) d u\right) .
\end{gathered}
$$

It remains to prove that

$$
\lim _{n \rightarrow \infty} G^{n+1}\left(\int_{0}^{t} \frac{(t-u)^{n}}{n !} F_{u}(x) d u\right)=\{0\} .
$$

There exists $M>0$ such that $\left\|F_{u}(x)\right\| \leq M$ for $u \in[0, t]$. Thus, by Lemma 9 , we see that

$$
\begin{aligned}
& \left\|G^{n+1}\left(\int_{0}^{t} \frac{(t-u)^{n}}{n !} F_{u}(x) d u\right)\right\| \\
& \leq\|G\|^{n+1} \int_{0}^{t} \frac{(t-u)^{n}}{n !}\left\|F_{u}(x)\right\| d u \\
& \leq\|G\|^{n+1} M \int_{0}^{t} \frac{(t-u)^{n}}{n !} d u
\end{aligned}
$$




$$
\begin{aligned}
& =\|G\|^{n+1} M \frac{t^{n+1}}{(n+1) !} \\
& =M \frac{(t\|G\|)^{n+1}}{(n+1) !} .
\end{aligned}
$$

Since $\lim _{n \rightarrow \infty} M \frac{(t\|G\|)^{n+1}}{(n+1) !}=0$, we have (6). Therefore, by (5),

$$
F_{t}(x) \subset \sum_{i=0}^{\infty} \frac{t^{i}}{i !} G^{i}(x) .
$$

By similar considerations the reader can prove that if $F_{t} \circ G=G \circ F_{t}$ for $t \geq 0$, then

$$
F_{t}(x)=\sum_{i=0}^{\infty} \frac{t^{i}}{i !} G^{i}(x)
$$

Lemma 13. Let $K$ be a closed convex cone with a nonempty interior in a Banach space. If $G: K \rightarrow c c(K)$ is a continuous additive multifunction and $F_{t}(x)=\sum_{i=0}^{\infty} \frac{t^{i}}{i !} G^{i}(x)$, then $t \mapsto F_{t}(x), x \in K$, is differentiable and

$$
D F_{t}(x)=G\left(F_{t}(x)\right), \quad x \in K, t \geq 0 .
$$

Proof. Let us fix $x \in K, t \geq 0$ and $h>0$. We have the equalities

$$
\frac{F_{t+h}(x)-F_{t}(x)}{h}=\lim _{n \rightarrow \infty} \sum_{i=0}^{n} \frac{1}{i !} \frac{(t+h)^{i}-t^{i}}{h} G^{i}(x)=\sum_{i=0}^{\infty} \frac{1}{i !} \frac{(t+h)^{i}-t^{i}}{h} G^{i}(x) .
$$

We will show that the series $\sum_{i=0}^{\infty} \frac{1}{i !} \frac{(t+h)^{i}-t^{i}}{h} G^{i}(x)$ is uniformly convergent. Let $h \in(0,1)$ and let $r$ be a positive number such that $t+1 \leq r$. Then

$$
\begin{aligned}
\left\|\frac{1}{i !} \frac{(t+h)^{i}-t^{i}}{h} G^{i}(x)\right\| & \leq \frac{1}{i !}\|G\|^{i}\|x\|\left((t+h)^{i-1}+t(t+h)^{i-2}+\cdots+t^{i-1}\right) \\
& \leq \frac{1}{i !}\|G\|^{i}\|x\| i r^{i-1} .
\end{aligned}
$$

Since the series $\sum_{i=1}^{\infty} \frac{(\|G\| r)^{i-1}}{(i-1) !}\|G\|\|x\|$ is convergent, it follows that the series $\sum_{i=1}^{\infty} \frac{1}{i !} \frac{(t+h)^{i}-t^{i}}{h} G^{i}(x)$ is uniformly convergent for $h \in(0,1)$. Therefore, we can write

$$
\begin{aligned}
\lim _{h \rightarrow 0^{+}} \frac{F_{t+h}(x)-F_{t}(x)}{h} & =\sum_{i=1}^{\infty} \frac{t^{i-1}}{(i-1) !} G^{i}(x) \\
& =G\left(\sum_{i=1}^{\infty} \frac{t^{i-1}}{(i-1) !} G^{i-1}(x)\right)=G\left(F_{t}(x)\right) .
\end{aligned}
$$


In a similar way we obtain

$$
\lim _{h \rightarrow 0^{+}} \frac{F_{t}(x)-F_{t-h}(x)}{h}=G\left(F_{t}(x)\right) .
$$

Whence

$$
D F_{t}(x)=G\left(F_{t}(x)\right) \quad \text { for } x \in K, t \geq 0 \text {. }
$$

Theorem 2. Let $K$ be a closed convex cone with a nonempty interior in a Banach space and let $G: K \rightarrow c c(K)$ be a continuous additive multifunction. Assume that $F_{t}(x)=\sum_{i=0}^{\infty} \frac{t^{i}}{i !} G^{i}(x)$ for $x \in K$ and $t \geq 0$. The family $\left\{F_{t}: t \geq\right.$ $0\}$ is an iteration semigroup if and only if

$$
F_{t} \circ G=G \circ F_{t}
$$

for $t \geq 0$.

Proof. Suppose that the family $\left\{F_{t}: t \geq 0\right\}$ is an iteration semigroup. By Lemma 13 this family is differentiable and $D F_{t}(x)=G\left(F_{t}(x)\right)$ for $x \in K$ and $t \geq 0$. On the other hand, from Theorem 1 , we have $D F_{t}(x)=F_{t}(G(x))$ for $x \in K$ and $t \geq 0$. Thus, $F_{t} \circ G=G \circ F_{t}$ for $t \geq 0$.

Now, we assume that (7) holds. Let $x \in K$ and $t \geq 0$. We observe that

$$
F_{t}(G(x))=\left(\sum_{i=0}^{\infty} \frac{t^{i}}{i !} G^{i}\right)(G(x)) \subset(I+t G)(G(x))+\sum_{i=2}^{\infty} \frac{t^{i}}{i !} G^{i+1}(x)
$$

and

$$
G\left(F_{t}(x)\right)=G\left(\sum_{i=0}^{\infty} \frac{t^{i}}{i !} G^{i}(x)\right)=G(x)+t G^{2}(x)+\sum_{i=2}^{\infty} \frac{t^{i}}{i !} G^{i+1}(x) .
$$

Since $F_{t}(G(x))=G\left(F_{t}(x)\right)$, we have

$$
G(x)+t G^{2}(x)+\sum_{i=2}^{\infty} \frac{t^{i}}{i !} G^{i+1}(x) \subset(I+t G)(G(x))+\sum_{i=2}^{\infty} \frac{t^{i}}{i !} G^{i+1}(x) .
$$

Thus,

$$
G(x)+t G^{2}(x) \subset(I+t G)(G(x)) .
$$

The inverse inclusion is obvious (see Lemma 7), therefore

$$
G(x)+t G^{2}(x)=(I+t G)(G(x)) .
$$

Similarly, we obtain

$$
G\left(F_{t}^{n}(x)\right)=F_{t}^{n}(G(x)) \text { for } n \in \mathbb{N},
$$


where $F_{t}^{n}(x)=\sum_{i=0}^{n} \frac{t^{i}}{i !} G^{i}(x)$. Thus,

$$
\begin{aligned}
F_{t}^{n}\left(F_{s}^{n}(x)\right) & =\left(\sum_{j=0}^{n} \frac{t^{j}}{j !} G^{j}\right)\left(\sum_{i=0}^{n} \frac{s^{i}}{i !} G^{i}(x)\right)=\sum_{j=0}^{n} \sum_{i=0}^{n} \frac{t^{j} s^{i}}{j ! i !} G^{i+j}(x) \\
& =\sum_{p=0}^{n} \sum_{q=0}^{p} \frac{t^{p-q} s^{q}}{(p-q) ! q !} G^{p}(x)+R_{n}=\sum_{p=0}^{n} \frac{(t+s)^{p}}{p !} G^{p}(x)+R_{n} \\
& =F_{t+s}^{n}(x)+R_{n},
\end{aligned}
$$

where $R_{n}=\sum_{p=n+1}^{2 n} \sum_{q=p-n}^{n} \frac{t^{p-q} s^{q}}{(p-q) ! q !} G^{p}(x)$. We see that

$$
\begin{aligned}
\left\|R_{n}\right\| & \leq \sum_{p=n+1}^{2 n} \sum_{q=p-n}^{n} \frac{t^{p-q} s^{q}}{(p-q) ! q !}\|G\|^{p}\|x\| \\
& =\sum_{p=n+1}^{2 n} \frac{1}{p !}(t+s)^{p}\|G\|^{p}\|x\| .
\end{aligned}
$$

Therefore

$$
\lim _{n \rightarrow \infty} R_{n}=\{0\}
$$

From Lemma 5 we get

$$
F_{t}\left(F_{s}(x)\right)=F_{t+s}(x) .
$$

In the end we show the relation between our condition and the condition in Ref. [10].

Theorem 3. Let $K$ be a closed convex cone with a nonempty interior in a Banach space. Assume that $G: K \rightarrow c c(K)$ is a continuous additive multifunction and $F_{t}(x)=\sum_{i=0}^{\infty} \frac{t^{i}}{i !} G^{i}(x)$ for $x \in K$ and $t \geq 0$.

1. If $F_{t} \circ G=G \circ F_{t}$ for $t \geq 0$, then $(I+t G) \circ G=G \circ(I+t G), t \geq 0$.

2. If $(I+t G) \circ G=G \circ(I+t G)$ and $0 \in G(x)$ for $t \geq 0, x \in K$, then $F_{t} \circ G=G \circ F_{t}$ for $t \geq 0$.

Proof. 1) It follows immediately from the proof of Theorem 2.

2) According to Theorem 1 in Ref. [10] the family $\left\{F_{t}: t \geq 0\right\}$ is an iteration semigroup. Thus, by Theorem 2 the equality

$$
F_{t} \circ G=G \circ F_{t}
$$

holds for all $t \geq 0$.

Example. Let $K=[0,+\infty)$ and let $\left\{F_{t}: t \geq 0\right\}$ be a family of multifunctions $F_{t}(x)=\left[x e^{a t}, x e^{b t}\right]$, as $0 \leq a \leq b$. Then this family is a differentiable iteration semigroup, $F_{t}(x)=\sum_{i=0}^{\infty} \frac{t^{i}}{i !} G^{i}(x)$, where $G(x)=[a x, b x]$ and $F_{t} \circ G=G \circ F_{t}$ for $t \geq 0$. 
Open Access. This article is distributed under the terms of the Creative Commons Attribution Noncommercial License which permits any noncommercial use, distribution, and reproduction in any medium, provided the original author(s) and source are credited.

\title{
References
}

[1] Hukuhara, M.: Intégration des application mesurables dont la valeur est un compact convexe. Funkcial. Ekvac. 10, 205-223 (1967)

[2] Nadler, S.B. Jr.: Multivalued contraction mappings. Pacific J. Math. 30, 475-488 (1969)

[3] Piszczek, M.: On multivalued cosine families. J. Appl. Anal. 13, 57-76 (2007)

[4] Piszczek, M.: Second Hukuhara derivative and cosine family of linear set-valued functions. Ann. Acad. Pead. Cracoviensis. Studia Math. 5, 87-98 (2006)

[5] Rådström, H.: An embedding theorem for spaces of convex sets. Proc. Am. Math. Soc. 3, 165-169 (1952)

[6] Smajdor, A.: Hukuhara's differentiable iteration semigroup of linear set-valued functions. Ann. Polon. Math. 83(1), 1-10 (2004)

[7] Smajdor, A.: Hukuhara's derivative and concave iteration semigrups of linear set-valued functions. J. Appl. Anal. 8, 297-305 (2002)

[8] Smajdor, A.: Increasing iteration semigroups of Jensen set-valued functions. Aequationes Math. 56, 131-142 (1998)

[9] Smajdor, A.: On a multivalued differential problem. Int. J. Bifur. Chaos Appl. Sci. Eng. 13, 1877-1882 (2003)

[10] Smajdor, A.: On concave iteration semigroups of linear set-valued functions. Aequationes Math. 75, 149-162 (2008)

[11] Smajdor, A.: On regular multivalued cosine families. Ann. Math. Sil. 13, 271-280 (1999)

[12] Smajdor, W.: Superadditive set-valued functions and Banach-Steinhaus Theorem. Rad. Mat. 3, 203-214 (1987)

\author{
Magdalena Piszczek \\ Institute of Mathematics \\ Pedagogical University \\ Podchorążych 2 \\ 30-084 Kraków \\ Poland \\ e-mail: magdap@ap.krakow.pl
}

Received: January 25, 2010

Revised: May 11, 2010 